\title{
Comments on Lone et al.: One-year prospective comparison of vaginal pessaries and surgery for pelvic organ prolapse using the validated ICIQ-VS and ICIQ-UI (SF) questionnaires
}

\author{
Kim van de Waarsenburg ${ }^{1} \cdot$ Astrid Vollebregt $^{2} \cdot$ Fred Milani $^{3}$. \\ Katrien Oude Rengerink ${ }^{4} \cdot$ Huub van der Vaart ${ }^{1}$
}

Received: 8 June 2015 / Accepted: 25 June 2015 / Published online: 4 August 2015

(C) The International Urogynecological Association 2015

\section{Dear Editor,}

We read with interest the publication by Lone et al. [1], where they prospectively compared the effectiveness of a vaginal pessary or surgery in women with symptomatic pelvic organ prolapse (POP). They concluded that "women can be reassured that by choosing a pessary as their first option for treatment of POP, the outcome at 1 year in terms of prolapse symptoms, urinary and bowel function, and quality of life can be as effective as that of surgery". In our opinion this conclusion is too strong for several reasons.

First, the analysis was not performed according to intention to treat: women who changed their decision after randomisation and switched from pessary therapy to surgery $(n=8)$ as well as women who discontinued the use of the pessary $(n=12)$ were excluded. By excluding women who are dissatisfied with pessary treatment, improvement after pessary therapy will be overestimated. Moreover, in total for $36 \%(104 / 287)$ of the women no change in score since base-

An author's reply to this comment is available at doi:10.1007/s00192015-2788-4.

Kim van de Waarsenburg

M.K.vandeWaarsenburg@umcutrecht.nl

1 Department of Obstetrics and Gynaecology, University Medical Centre, Utrecht, The Netherlands

2 Department of Obstetrics and Gynaecology, Spaarne Hospital, Hoofddorp, The Netherlands

3 Department of Obstetrics and Gynaecology, Reinier de Graaf Hospital, Delft, The Netherlands

4 Department of Obstetrics and Gynaecology, Academic Medical Centre, Amsterdam, The Netherlands line could be calculated at 1 year. Therefore, selective loss to follow-up cannot be excluded.

Second, both pessary therapy and surgery showed statistically significant improvement in prolapse symptoms, urinary and bowel function, and quality of life. Although the decline in bothersome symptoms is described, no interpretation of when an improvement is considered relevant for the patient is given. In the surgery group there is more decline for almost all symptoms, but these differences are not statistically significant. However, the on average much smaller decline within a woman is statistically significant and therefore called important. Given the relatively small sample size of the study, a potentially clinically relevant difference might not be detected, but can also not be excluded.

In our opinion, the cost-effectiveness of a primary treatment strategy of pessary or surgery for moderate to severe prolapse still deserves further evaluation [2], preferably in a well-powered randomised trial. We recently started the PEOPLE study in the Netherlands, which is a multicentre pragmatic cohort study with an embedded randomised trial comparing the (cost) effectiveness of pessary therapy versus surgery (NTR 4883). We expect results in 2017.

Conflicts of interest None.

\section{References}

1. Lone F, Thakar R, Sultan AH (2015) One-year prospective comparison of vaginal pessaries and surgery for pelvic organ prolapse using the validated ICIQ-VS and ICIQ-UI (SF) questionnaires. Int Urogynecol J

2. Bugge C, Adams EJ, Gopinath D et al (2013) Pessaries (mechanical devices) for pelvic organ prolapse in women. Cochrane Database Syst Rev 2:CD004010 